\title{
Influencia de las características y técnicas constructivas en las propiedades mecánicas de vigas laminadas encoladas de madera de Pinus caribaea var. hondurensis
}

\author{
Influence of the growth characteristics and fabrication \\ techniques on the mechanical properties of Pinus caribaea \\ var. hondurensis timber glued-laminated beams
}

\author{
Pablo Ninin 1 , Wilver Contreras-Miranda 2 y Styles W. Valero 3
}

\begin{abstract}
RESUMEN
El presente trabajo es la culminación de un amplio espectro de investigaciones realizadas en el Laboratorio Nacional de Productos Forestales, Mérida, Venezuela, centradas en valorar las potencialidades de la madera de Pinus caribaea var. hondurensis en la elaboración de vigas laminadas encoladas con calidad estructural. Este estudio permitió determinar, a partir de la inferencia de los análisis estadísticos, la posible influencia de las características de crecimiento y de las uniones longitudinales "dentadas" finger joint, en las propiedades mecánicas: esfuerzo en el límite de proporcionalidad (ELP), módulo de ruptura (MOR) y módulo de elasticidad (MOE) de dichas vigas laminadas. Se logró determinar, en orden de importancia, qué defectos son relevantes para estas propiedades mecánicas, llegando a conclusiones y recomendaciones muy concretas que llaman a la reflexión a los fabricantes venezolanos de vigas laminadas encoladas, para la mejora continua de sus procesos, productos y servicios, a fin de poder seguir manteniendo la oferta de productos estructurales seguros y de alta calidad en los niveles de resistencia mecánica.
\end{abstract}

PALABRAS CLAVE:

Análisis estadísticos, esfuerzo en el límite de proporcionalidad, esfuerzos de diseño, módulo de elasticidad, módulo de ruptura.

\begin{abstract}
This paper is the culmination of a wide range of researches carried out at the Laboratorio Nacional de Productos Forestales, Mérida, Venezuela, focused on assessing the potentials of the Pinus caribaea var. hondurensis timber for the production of structural quality glued-laminated beams. Hence, the study allows determining, from the statistical analysis inference, the growth characteristics and fabrication techniques, the possible influences on the mechanical properties of glued laminated beams, especially the finger joints, with respect to the stress at Proportional Limit (ELP), Modulus of Rupture (MOR) and Modulus of elasticity (MOE). From the results, the influence of those defects can
\end{abstract}

Facultad de Ciencias Forestales y Ambientales. Laboratorio Nacional de Productos Forestales (LNPF). Sección de Aserrado y Trabajabilidad. Universidad de Los Andes, Mérida, Venezuela. ninin@ula.ve.

2 Centro de Estudios Forestales y Ambientales de Posgrado (CEFA -ULA). Facultad de Ciencias Forestales y Ambientales. Laboratorio Nacional de Productos Forestales (LNPF). Universidad de Los Andes, Mérida, Venezuela. wilvercontrerasmiranda@yahoo.es; wilconmi@doctor.upv.es.

3 Facultad de Ciencias Forestales y Ambientales. Laboratorio Nacional de Productos Forestales (LNPF). Sección de Ensayos. Universidad de Los Andes, Mérida, Venezuela. styles@ula.ve. 
be determined in order of importance, which allows to get very concrete conclusions and recommendations to make the glued-laminated beam Venezuelan manufacturers reflect about the continuous improvement of their processes, products and services with the aim of keeping the supply of high quality and reliable structural products.

KEY WORDS:

Statistical analysis, stress at proportional limit, design parameters, modulus of elasticity, modulus of rupture.

\section{INTRODUCCIÓN}

La calidad de la madera como material orgánico anisotrópico, ya sea en su condición de madera sólida o como un producto forestal de valor agregado y alto valor agregado, caso de la madera laminada encolada, depende de toda una suma de factores intrínsecos y extrínsecos propios de la anatomía del material, niveles de calidad de los procesos de transformación para la obtención y manufactura del producto forestal final, condiciones de uso y los esfuerzos a los que estará sometido el mismo (Contreras et al., 2005; Cloquell et al., 2007).

Desde el punto de vista de sus defectos anatómicos y de las propiedades mecánicas, la calidad de la madera es evaluada en función de los defectos que ésta contenga, principalmente las grietas, rajaduras, desviación de la fibra, tamaño de nudos, acebolladuras, ataques de agentes xilófagos y otros más específicos como el grosor de la pared celular, la presencia o no de parénquima, presencia de extractivos o contenido de humedad (León y Espinosa de Pernía, 2002; Valero, 2001). La influencia de estos defectos, en el caso de la madera sólida o madera laminada encolada con fines estructurales, se considera mediante el uso de normas de clasificación que varian mucho, pudiendo ser visuales (los más usados) o mecánicos destructivos y no destructivos (Smulski, 1997). De ahí que el desarrollo de nuevos productos forestales con aplicaciones estructurales se orienta a la eliminación de la influencia de los defectos en la resistencia mecánica de la madera. La madera laminada, microlaminada o el tablero contrachapado limitan la influencia de sucesivos defectos (Ross, 1985; Ross et al., 1996).

Por consiguiente, la proyección de uso de la madera del Pinus caribaea var. hondurensis de las plantaciones forestales del oriente de Venezuela en la manufactura industrial, ya sea en productos forestales de madera sólida o productos forestales derivados, obliga de manera inmediata a la profundización de las investigaciones científicas y tecnológicas para la mejora continua del diseño industrial de estos productos.

\section{OBJETIVOS}

Estudiar la influencia de los defectos o características de crecimiento de la madera y de la técnica de uniones longitudinales "dentadas" o de finger joint, en las propiedades mecánicas de las vigas laminadas encoladas, fabricadas con madera de Pinus caribaea var. hondurensis y adhesivo isocianato dimetil (MDI). A partir de este estudio se podrá llegar a inferir técnicamente la caracterización, por orden de importancia, de los posibles factores negativos que más perjudican en alcanzar los altos estándares exigidos según las normas internacionales, a los productos laminados con calidad estructural manufacturados en Venezuela.

\section{METODOLOGÍA}

La investigación se realizó en las instalaciones del Laboratorio Nacional de Productos Forestales Sección de Ensayos de la Universidad de Los Andes (Mérida, Venezuela). La misma se desarrolló a partir 
de los resultados obtenidos por Contreras et al. (2007), en la determinación de los esfuerzos de diseño de las vigas laminadas encoladas de Pinus caribaea var. hondurensis y adhesivo MDI. La tabla 1 expone los resultados obtenidos del estudio previo y la descripción de las características dimensionales de las vigas. El presente estudio se centra en el análisis detallado de 18 vigas, de las cuales nueve fueron manufacturadas a partir del uso de la técnica de unión finger joint identificadas como FJ, y las otras nueve fueron fabricadas con láminas (tablas/ tablones) completas, identificadas como EC. La industria fabricante es la única de este ramo, hasta el presente, en Venezuela, encontrándose localizada en la zona industrial de Matanzas, de la Ciudad de Puerto Ordaz, Estado Bolívar.

Una vez recibidas las vigas en las instalaciones del laboratorio, se procedió a su identificación con números que van de 1 hasta el 18 (Tabla 1). Posteriormente cada viga fue analizada morfológicamente según los aspectos anatómicos (color, tamaño y localización de nudos en cada viga, dirección de la fibra, presencia de defectos por pudrición); aspectos técnicos según lo definido por Freas y Selbo (1954), de la manufactura de las vigas (calidad física de las líneas de cola, aserrado-labrado mecanizado para la conformación de las láminas o tablas/ tablones de madera-uniones dentadas). Las dimensiones nominales de las láminas que conforman cada una de las vigas a partir de tablas son de 2,5 centímetros y de los tablones 5 centímetros. Las dimensiones nominales de las vigas las exponen detalladamente Contreras et al. (2007). La determinación de los esfuerzos de diseño se realizó a partir de los ensayos de flexión estática de acuerdo con la Norma ASTM D-198-84. La información se reportó de manera detallada en planillas descriptivas y gráficas. El análisis de estadística descriptiva, análisis de varianza y análisis multiva- riado de componentes principales se desarrolló a partir de los valores expuestos en la tabla 1 que fueron introducidos, entre otros valores, en la base de datos desarrollada con el software Minitab versión 12.

\section{RESULTADOS Y DISCUSIÓN}

\section{Estadística descriptiva de las propie- dades mecánicas de las vigas lami- nadas de Pinus caribaea var. hondu- rensis}

En las tablas 2 y 3 se observan las estadísticas descriptivas de las propiedades mecánicas de Deformación $(\mathrm{mm})$ en el Esfuerzo al Límite Proporcional, Esfuerzo al Límite Proporcional (ELP) (MPa), Módulo de Ruptura (MOR - MPa) y Módulo de Elasticidad (MOE-MPa), tanto para vigas compuestas por elementos unidos por medio de la técnica de finger joint, como por aquellas compuestas por elementos completos.

A partir de los resultados de la estadística descriptiva se puede afirmar que las vigas elaboradas con finger joint presentan menores valores promedio en las propiedades mecánicas Deformación ( $\mathrm{mm}$ ) en el Esfuerzo al límite Proporcional (MPa) y Módulo de Ruptura (MPa) que los promedios obtenidos en vigas elaboradas con láminas completas.

Se destaca que el Módulo de Elasticidad (MPa) es ligeramente mayor en su valor promedio para vigas elaboradas con finger joint que para vigas elaboradas con láminas completas. Considerando la desviación estándar como medida de dispersión de los valores de las propiedades mecánicas en estudio, se destaca que los valores son mayores para vigas elaboradas con láminas completas que para vigas elaboradas con finger joint. 


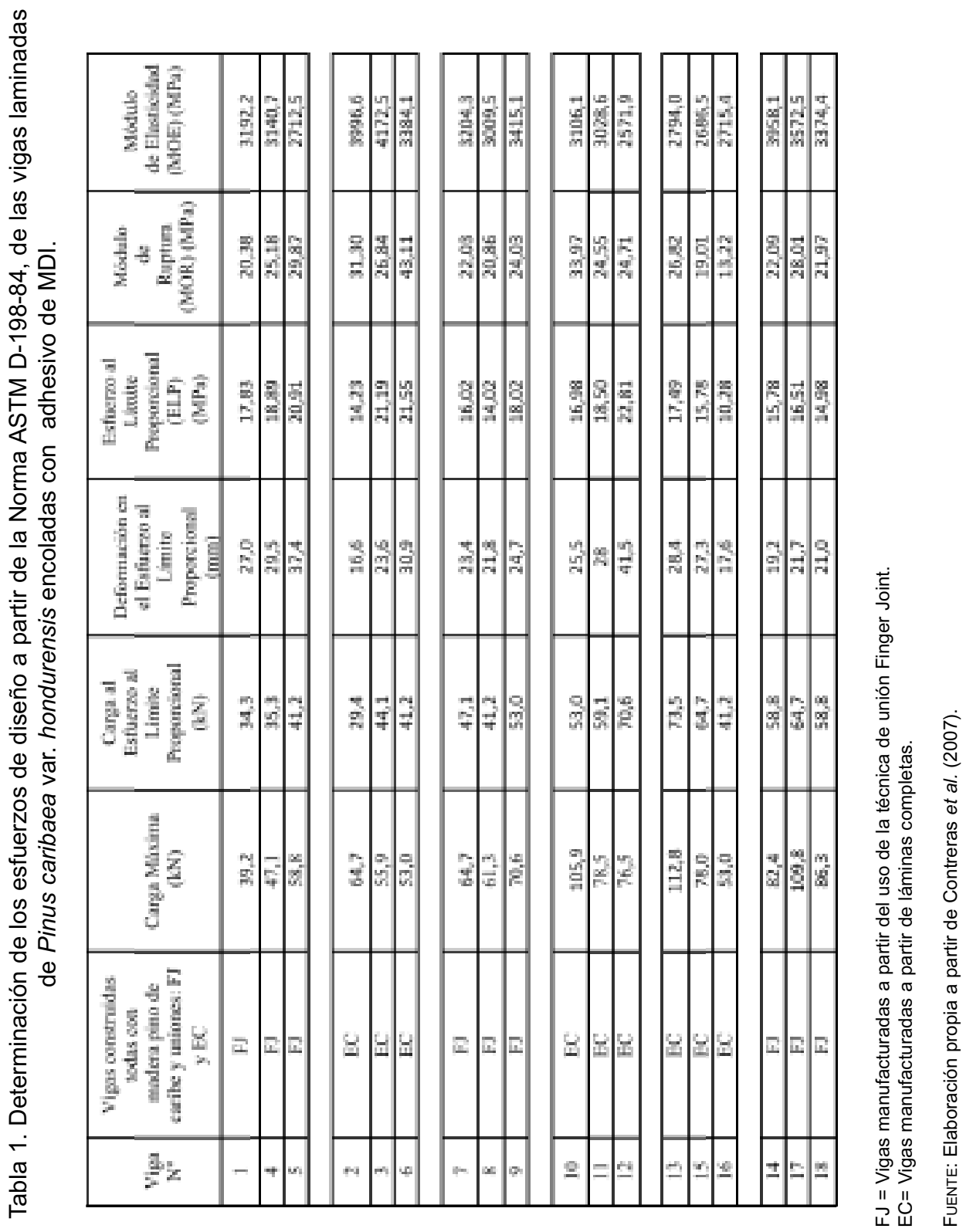


Tabla 2. Estadística descriptiva de las propiedades mecánicas de vigas laminadas compuestas por elementos unidos por finger joint.

\begin{tabular}{lccc}
\hline Propiedad mecánica & $\mathrm{n}$ & Promedio & $\begin{array}{c}\text { Desviación } \\
\text { Estándar }\end{array}$ \\
\hline Deformación al Limite Proporcional (mm) & 9 & 27,3 & 5,6 \\
Esfuerzo al limite proporcional (ELP) (MPa) & 9 & 17,61 & 2,37 \\
Módulo de ruptura (MOR) (MPa) & 9 & 23,73 & 3,53 \\
Módulo de elasticidad (MOE) (MPa) & 9 & 3112,4 & 235,7 \\
\hline
\end{tabular}

Tabla 3. Estadística descriptiva de las propiedades mecánicas de vigas laminadas compuestas por elementos completos.

\begin{tabular}{lccc}
\hline Propiedad Mecánica & $\mathrm{n}$ & Promedio & $\begin{array}{c}\text { Desviación } \\
\text { Estándar }\end{array}$ \\
\hline Deformación en el Esfuerzo al Límite Proporcional (mm) & 9 & 27,7 & 8,3 \\
Esfuerzo al límite proporcional (ELP) (MPa) & 9 & 19,21 & 3,24 \\
Módulo de ruptura (MOR) (MPa) & 9 & 30,42 & 7,50 \\
Módulo de elasticidad (MOE) (MPa) & 9 & 3376,6 & 609,9 \\
\hline
\end{tabular}

Tabla 4. Análisis multivariados de componentes principales, variación explicada para la propiedad mecánica Deformación $(\mathrm{mm})$ en el Esfuerzo al Límite Proporcional, considerando variables de manufactura y anatómicas en vigas laminadas ( $n=18$ vigas).

\begin{tabular}{lccc}
\hline Aspectos de crecimiento y manufactura & Valor propio & Proporción & Acumulada \\
\hline Número de Nudos & 2,5623 & 0,512 & 0,512 \\
Número de Uniones Finger Joint & 1,7401 & 0,348 & 0,860 \\
Tipo de Viga & 0,4102 & 0,082 & 0,943 \\
Leño Juvenil & 0,2670 & 0,053 & 0,996 \\
Deformación (mm) en el Esfuerzo al Límite Proporcional & 0,0204 & 0,004 & 1,000 \\
\hline
\end{tabular}

Tabla 5. Análisis multivariado de componentes principales, variación explicada para la propiedad mecánica Esfuerzo al Límite Proporcional (MPa), considerando variables de manufactura y anatómicas en vigas laminadas ( $n=18$ vigas).

\begin{tabular}{lccc}
\hline Aspectos de crecimiento y manufactura & Valor propio & Proporción & Acumulada \\
\hline Número de Nudos & 2,4121 & 0,482 & 0,482 \\
Número de Uniones Finger Joint & 1,6775 & 0,335 & 0,818 \\
Tipo de Viga & 0,4963 & 0,099 & 0,917 \\
\% Leño Juvenil & 0,3926 & 0,079 & 0,996 \\
Esfuerzo al Límite proporcional (MPa) & 0,0216 & 0,004 & 1,000 \\
\hline
\end{tabular}




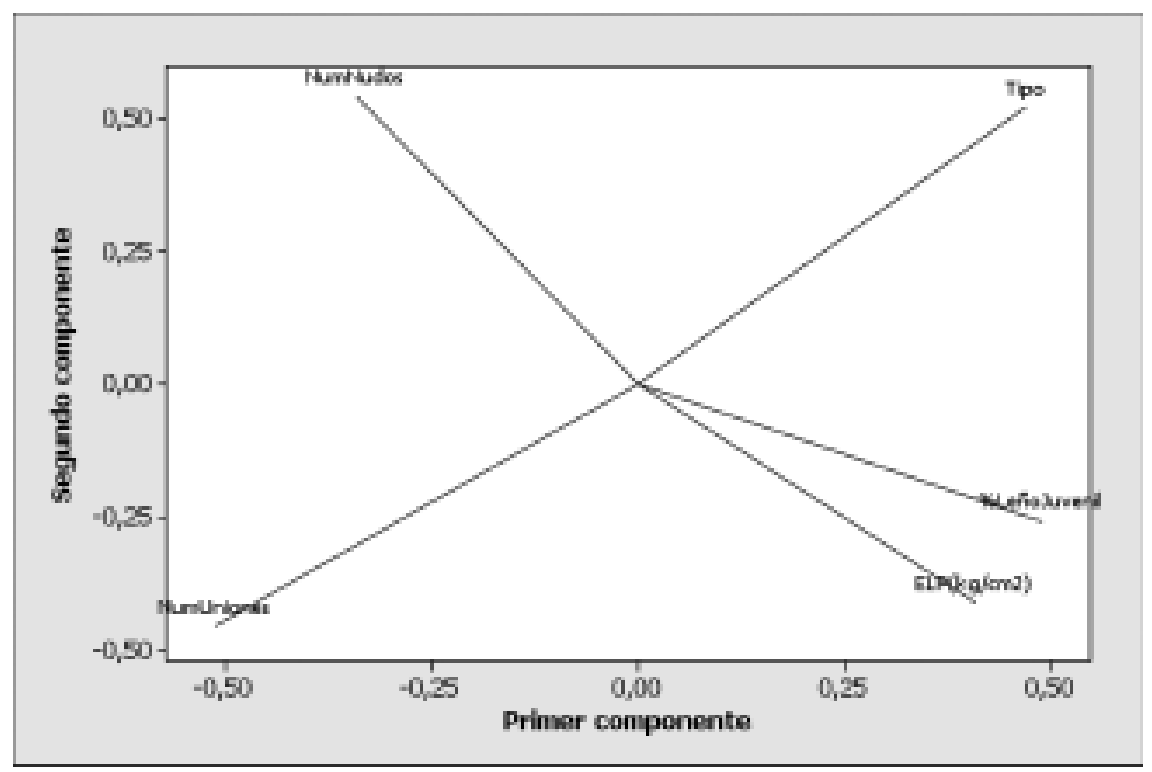

Figura 1. Relación de variables de manufactura y anatómicas, con respecto a la propiedad mecánica Deformación $(\mathrm{mm})$ en el Esfuerzo al Límite Proporcional, mediante el resultado gráfico de análisis multivariado de componentes principales.

Entre las vigas elaboradas con finger joint y las vigas elaboradas con láminas completas no existen diferencias estadísticas significativas a un $95 \%$ de confiabilidad, para las propiedades mecánicas en estudio. Esta afirmación se fundamenta en los resultados de los análisis de varianza de una vía, diferencia de medias de Tukey y Fisher, considerando como variable dependiente el tipo de viga $y$ como variables dependientes la Deformación $(\mathrm{mm})$ en el Esfuerzo al Límite Proporcional (MPa), Módulo de Ruptura (MPa) y Módulo de Elasticidad (MPa). No existen diferencias estadísticas significativas en las propiedades mecánicas causadas por el tipo de manufactura de vigas en estudio. Los mayores valores de desviación estándar, de las propiedades mecánicas correspondientes a vigas elaboradas con láminas completas, sugieren que el control de calidad, para valores admisibles de carga de refe- rencia, generará mayores dificultades que para vigas elaboradas con finger joint.

\section{Análisis multivariado de componentes principales de las propiedades mecá- nicas de las vigas laminadas de Pinus caribaea var. hondurensis}

El análisis de componentes principales (ACP) es una técnica utilizada para reducir la dimensionalidad de un conjunto de datos. Intuitivamente la técnica sirve para determinar el número de factores subyacentes explicativos tras un conjunto de datos que expliquen la variabilidad de dichos datos. Se emplea sobre todo en análisis exploratorio de datos y para construir modelos predictivos.

La variación de la propiedad mecánica Deformación (mm) en el Esfuerzo al Límite Proporcional en vigas laminadas es expli- 


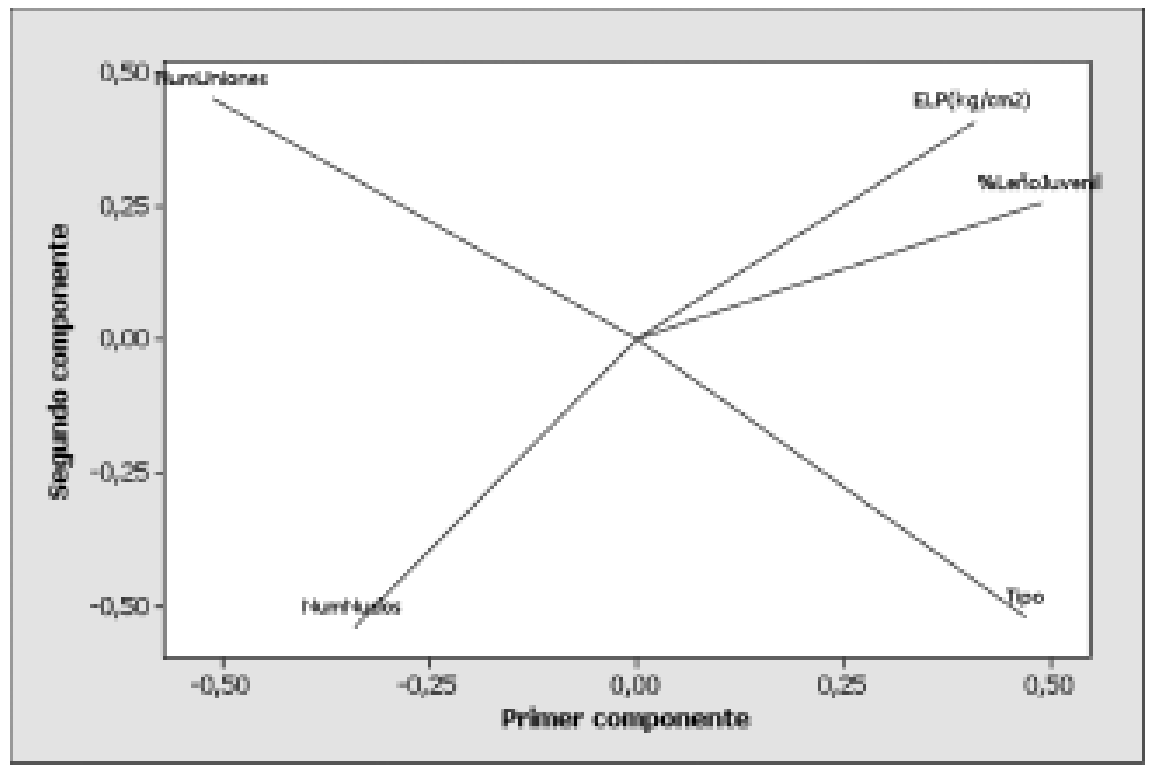

Figura 2. Relación de variables de manufactura y anatómicas, con respecto a la propiedad mecánica Esfuerzo al límite proporcional (MPa), mediante el resultado gráfico de análisis multivariado de componentes principales.

cada en $51,2 \%$ por la variable número de nudos, $34,8 \%$ por el número de uniones, $8,2 \%$ por el tipo de viga y apenas $5,3 \%$ por el leño juvenil (Tabla 4). En la figura 1 se observa la relación de variables de manufactura y anatómicas, con respecto a la Deformación $(\mathrm{mm})$ en el Esfuerzo al Límite Proporcional, donde se destaca que la propiedad mecánica en cuestión resultó con un comportamiento indirectamente proporcional con la variable anatómica número de nudos, siendo esta variable la que explica la mayor variación. Es decir, en la medida que se incrementa el número de nudos en una viga laminada los valores de Deformación (mm) en el Esfuerzo al Límite Proporcional se hacen menores. La posición de los vectores de las variables de manufactura Tipo de Viga y Número de uniones, con respecto a la posición del vector de la variable mecánica en cuestión, expresa una mínima incidencia en su magnitud.
En el tabla 5 se observa la explicación de la variación propiedad mecánica Esfuerzo al Límite Proporcional (MPa) en vigas laminadas. La variable Número de Nudos explica $48,2 \%$ de la variación de la propiedad mecánica en cuestión, seguida por $33,5 \%$ de la variable Número de Uniones, siendo apenas de $9,9 \%$ y $7,9 \%$ de Tipo de Viga y \%Leño Juvenil, respectivamente. En la figura 2 se destaca un patrón similar al observado para la variable de propiedad mecánica Deformación $(\mathrm{mm})$ en el Esfuerzo al Límite Proporcional.

En atención a las propiedades mecánicas, Deformación ( $\mathrm{mm}$ ) y Esfuerzo al Límite Proporcional ( $\mathrm{MPa})$, una se corresponde con la otra, vale decir, es la deformación o deflexión $(\mathrm{mm})$ de la viga como respuesta a una carga $(\mathrm{MPa})$ límite que permite mantener sus propiedades elásticas. Si se aplica una carga (MPa) 
Tabla 6. Análisis multivariado de componentes principales, variación explicada para la propiedad mecánica Módulo de Ruptura (MPa), considerando variables de manufactura y anatómicas en vigas laminadas ( $n=18$ vigas).

\begin{tabular}{lccc}
\hline Aspectos de crecimiento y manufactura & Valor propio & Proporción & Acumulada \\
\hline Número de Nudos & 2,2775 & 0,456 & 0,456 \\
Número de Uniones Finger Joint & 1,3886 & 0,278 & 0,733 \\
Tipo de Viga & 1,0546 & 0,211 & 0,944 \\
\% Leño Juvenil & 0,2572 & 0,051 & 0,996 \\
Esfuerzo al Limite proporcional (MPa) & 0,0220 & 0,004 & 1,000 \\
\hline
\end{tabular}

Tabla 7. Análisis multivariado de componentes principales, variación explicada para la propiedad mecánica Módulo de Elasticidad (MPa), considerando variables de manufactura y anatómicas en vigas laminadas ( $n=18$ vigas).

\begin{tabular}{lccc}
\hline Aspectos anatómicos y manufactura & Valor propio & Proporción & Acumulada \\
\hline Número de Nudos & 2,4028 & 0,481 & 0,481 \\
Número de Uniones Finger Joint & 1,6001 & 0,320 & 0,801 \\
Tipo de Viga & 0,5915 & 0,118 & 0,919 \\
\% Leño Juvenil & 0,3841 & 0,077 & 0,996 \\
Esfuerzo al Límite proporcional (MPa) & 0,0215 & 0,004 & 1,000 \\
\hline
\end{tabular}

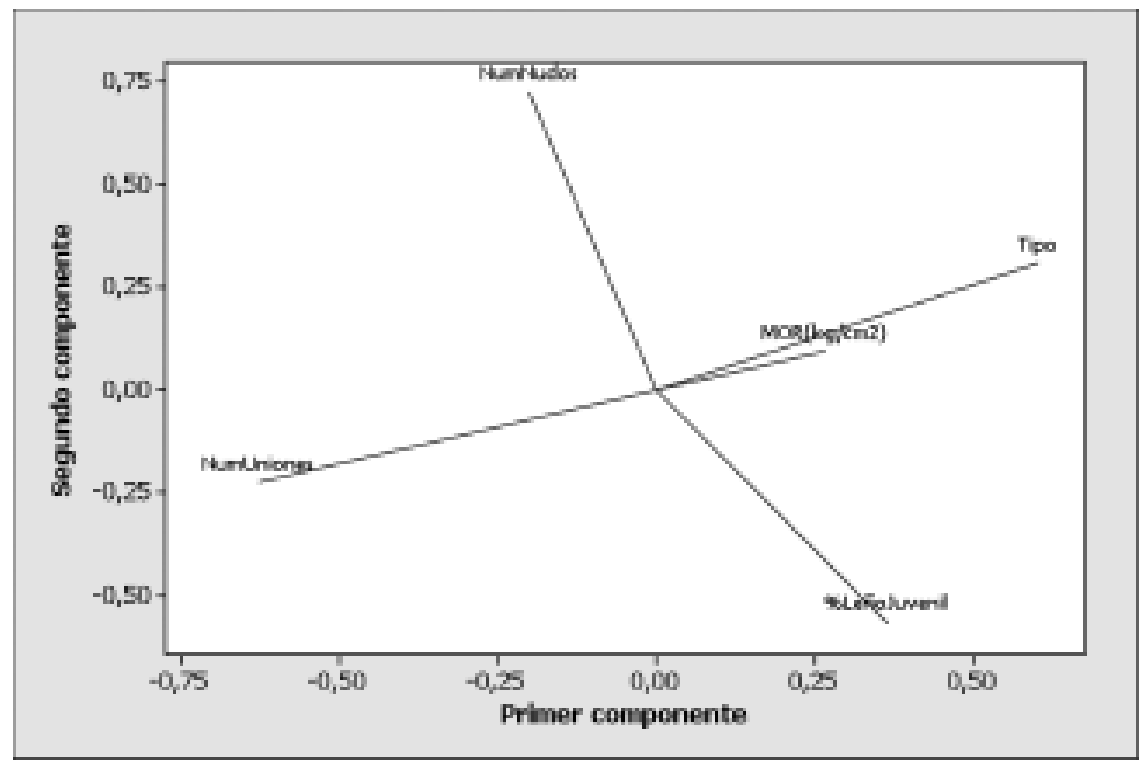

Figura 3. Relación de variables de manufactura y anatómicas, con respecto a la propiedad mecánica Módulo de Ruptura (MPa), mediante el resultado gráfico de análisis multivariado de componentes principales. 
mayor, la viga presentará una deformación permanente. Para ambas propiedades mecánicas la variable de mayor incidencia se corresponde con el Número de Nudos, representando este defecto discontinuidad o interrupción en los tejidos de madera que conforman una viga y que soportan una carga específica. Una viga con pocos nudos tendrá una mayor magnitud de deflexión frente a una carga específica, puesto que se trata de un elemento más homogéneo y menos rígido frente a la aplicación de cargas.

Las variables de manufactura y anatómicas se relacionaron con la variable Módulo de Ruptura (MOR) (MPa) mediante el análisis de componentes principales, del cual se destaca que $45,6 \%$ de la variación se debe al Número de Uniones, $27,8 \%$ al Tipo de Viga, $21,1 \%$ para Número de Nudos y apenas $5,1 \%$ por causa del \%Leño Juvenil. En la figura 3 se observa la relación de variables de manufactura y anatómicas, con respecto al Módulo de Ruptura (MOR), donde se destaca que la propiedad mecánica resultó con un comportamiento indirectamente proporcional con la variable de manufactura Número de Uniones, siendo esta variable la que explica 45,6\% de la variación del Módulo de Ruptura (MOR) (MPa). De la posición de vectores se deduce que en la medida que se incrementa el Número de Uniones, los valores de Módulo de Ruptura (MOR) (MPa) se hacen menores. La posición de los vectores de las variables anatómicas Número de Nudos y el \%Leño Juvenil, con respecto a la posición del vector de la variable mecánica en cuestión, expresan una mínima incidencia en su magnitud.

El Módulo de Ruptura (MPa) expresa la carga necesaria para producir la ruptura de la viga por falla en flexión (deformación elástica, deformación permanente o plástica, ruptura). En la medida que el número de uniones finger joint se incrementan en los elementos constitutivos de las vigas laminadas, la carga necesaria para generar su ruptura o falla por flexión en el Módulo de Ruptura (MOR), es menor. Es decir, que en la medida que se minimicen el número de uniones finger joint entre la luz de carga, se incrementará la resistencia de la viga. La unión de finger joint es menos elástica que la madera, y es más rígida que la madera sin unión, es por ello que el incremento de número de uniones determina que el valor de la deformación elástica sea muy cercano al valor de ruptura. Una viga sin uniones tiende a presentar valores de deformación elástica notablemente menores que su correspondiente valor de ruptura.

La variación propiedad mecánica, Módulo de Elasticidad (MPa) en vigas laminadas es explicada en $48,1 \%$ por la variable Leño (madera) Juvenil, 32,0\% por el Número de Nudos, $11,8 \%$ por el número de Uniones y apenas $7,7 \%$ por el leño juvenil (Tabla 7). En la figura 4 se observa la relación de variables de manufactura y anatómicas, con respecto al Módulo de Elasticidad (MPa), donde se destaca que la propiedad mecánica en cuestión resultó con un comportamiento indirectamente proporcional con la variable anatómica \%Leño Juvenil, siendo esta variable la que explica la mayor variación.

Es decir, en la medida que se incrementa el \%Leño Juvenil en una viga laminada los valores de Módulo de Elasticidad (MPa) se hacen menores. La posición de los vectores de las variables de manufactura Tipo de Viga y Número de uniones, con respecto a la posición del vector de la variable mecánica en cuestión, expresan una mínima incidencia en su magnitud. 


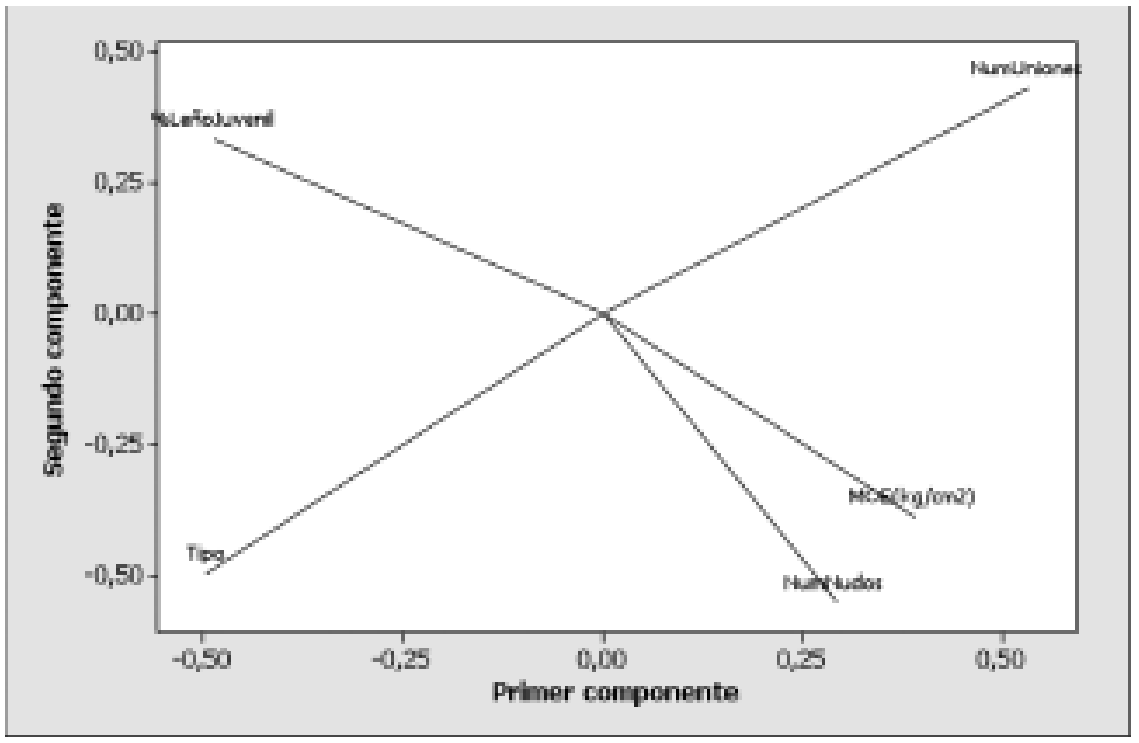

Figura 4. Relación de variables de manufactura y anatómicas, con respecto a la propiedad mecánica Módulo de Elasticidad (MOE) (MPa), mediante el resultado gráfico de análisis multivariado de componentes principales.

El Módulo de Elasticidad (MPa) indica la capacidad que tiene un material para recuperar su forma y tamaño original después de remover los esfuerzos, es decir, es una medida de resistencia del material. La variable anatómica que repercute de manera más contundente en la capacidad de una viga laminada de soportar cargas sin deformarse luego de ser retirada la carga se corresponde con el Leño Juvenil presente. La madera juvenil del Pinus caribaea var. hondurensis de la Orinoquía Venezolana se caracteriza por presentar menor densidad, menor número de anillos de crecimiento (Ninin, 2007), y menores longitudes de traqueidas que la madera adulta (Trejo, 2006). Este conjunto de variables afecta la resistencia mecánica de la madera juvenil.

\section{CONCLUSIONES}

El presente estudio permite concluir que las propiedades mecánicas de las vigas laminadas encoladas de Pinus caribaea var. hondurensis de la Orinoquía Venezolana, se ven afectadas según los defectos evaluados, en el siguiente orden de importancia:

1. Número de Nudos afecta la capacidad de deformación o deflexión ( $\mathrm{mm}$ ) de la viga.

2. Número de Uniones afecta los valores de Módulo de Ruptura (MOR) (MPa).

3. Porcentaje de Leño Juvenil afecta los valores del Módulo de Elasticidad (MPa)

De acuerdo con los objetivos planteados, la determinación de la caracterización por niveles de importancia de los defectos en las vigas laminadas encoladas de Pinus caribaea var. hondurensis, permiten recomendar a los fabricantes de este tipo de vigas que se debe tener presente la mejora en los procesos de fabricación, especialmente en la técnica de finger joint, la cual 


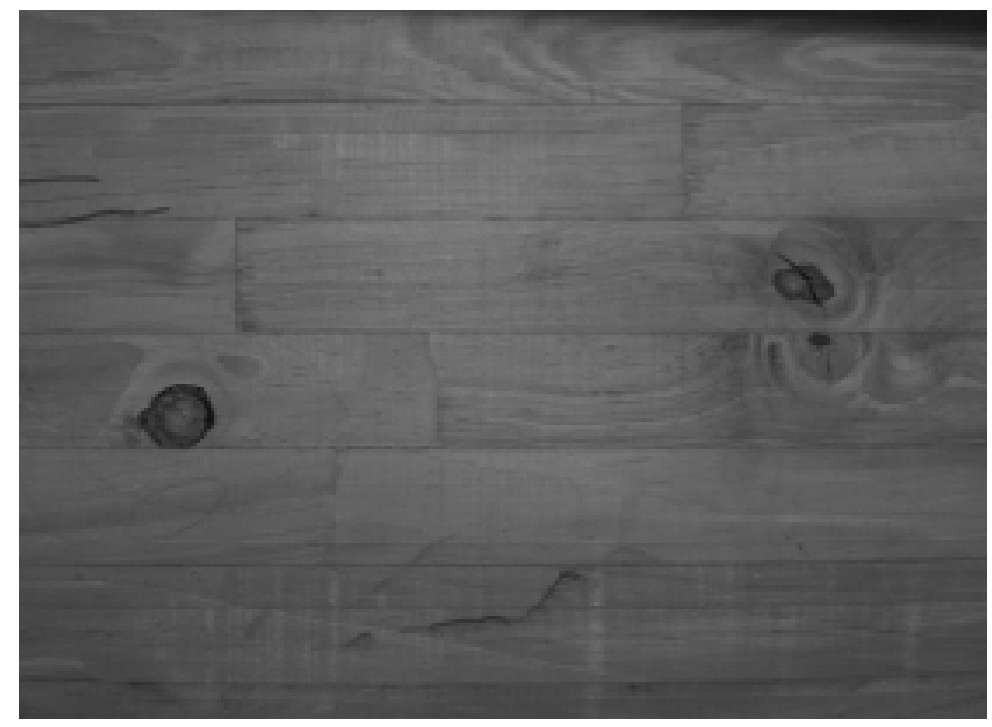

Figura 5. Apreciación visual y técnica de la cantidad y tamaño de los nudos en las láminas de madera que conforman una de las vigas laminadas realizadas con la técnica de finger joint. Se puede apreciar el mal uso de esta técnica al quedar, en este caso de estudio, las uniones muy cercanas entre sí, lo cual ocasiona la falla y una menor resistencia del elemento laminado al momento del ensayo.

(Foto: Wilver Contreras Miranda)

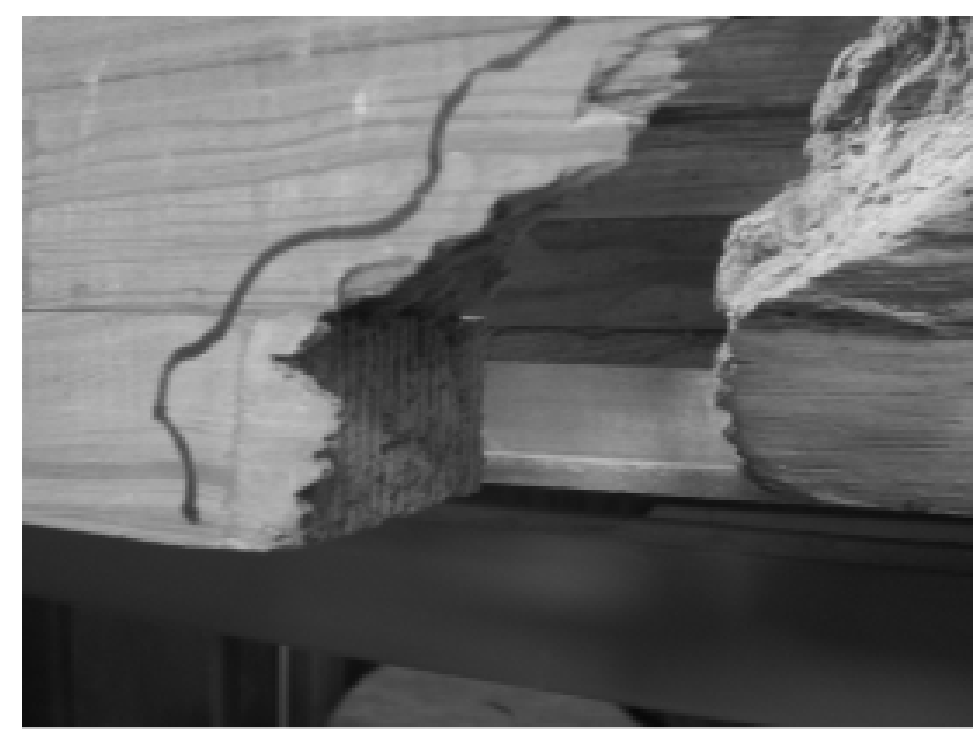

Figura 6. Apreciación visual de una unión finger joint ubicada en la zona de tracción, de una de las vigas estudiadas, contribuyendo a ocasionar la falla del elemento laminado al momento del ensayo. (Foto: Wilver Contreras Miranda) 
viene siendo mal empleada según los criterios de uso formulados, entre otros, por Freas y Selbo (1954), American Association of State Highway Officials (1973), American Institute of Timber Construction (1984), American Institute of Timber Construction (1985), Contreras y Owen de Contreras (1999) o Contreras et al. (2000).

La mejora del uso de la técnica permitirá aumentar los valores en los esfuerzos de diseño y una mejor proyección de seguridad en el uso de las piezas estructurales en las edificaciones. Respecto a los porcentajes en la presencia de madera de leño juvenil, inherente a la calidad anatómica de la madera de Pinus caribaea var. hondurensis, se recomienda no incluir piezas con importantes porcentajes de este defecto y de tal forma obtener una calidad de uso estructural de forma consistente. Para evitar de manera contundente la participación del leño juvenil, se recomienda para la obtención de piezas aserradas que conforman las vigas, la aplicación del reaserrado con la conicidad, vale decir, que siga la conicidad de las trozas, de tal forma que al encajonar el leño juvenil en una cuña central se evitará que éste se mezcle con madera aserrada madura en una misma pieza (Ninin, 2007).

En referencia a los nudos, es inherente la cantidad de éstos en el fuste del árbol de Pinus caribaea var. hondurensis de la Orinoquía Venezolana, se tiene una gran presencia y cercanía de los verticilos de nudos. Esto afecta notablemente la calidad estructural de esa madera.

Se recomienda desarrollar nuevas investigaciones que permitan evidenciar y ponderar el efecto del porcentaje de leño juvenil en piezas que conforman las vigas laminadas encoladas de Pinus caribaea var. hondurensis, siendo para ello imprescindible controlar o congelar el efecto del resto de defectos sobre las resistencias mecánicas, tales como nudos y número de uniones de finger joint.

\section{AGRADECIMIENTOS}

Se agradece al Técnico de Laboratorio Rolando Betancourt de la Sección de Ensayos del LNPF-ULA-MPPA su apoyo para la realización de las pruebas de laboratorio.

\section{REFERENCIAS}

American Association of State Highway Officials. 1973. Construction manual for highway bridges and incidental structures. Washington, DC: American Association of State Highway Officials. 66 pp.

American Institute of Timber Construction. 1984. Typical construction details. AITC 104-84. Englewood, CO: American Institute of Timber Construction. 32 pp.

American Institute of Timber Construction. 1985. Timber construction manual. $3 d$ ed. New York: John Wiley and Sons, Inc. 836 pp.

Cloquell, V., W. Contreras y M. Owen de Contreras. 2007. La madera y los productos forestales en sistemas estructurales. Aspectos técnicos y medioambientales. Editorial Fundación Politécnica Antiguos Alumnos. Universidad Politécnica de Valencia. Valencia, España. 280 pp.

Contreras, W., M. Owen de C., F. Rosso y Y. Contreras. 2000. El desarrollo de la tecnología de la madera laminada, y sus perspectivas de uso en Venezuela. Revista Forestal Latinoamericana. IFLA. Mérida, Venezuela. Vol. 15, 26(00):17 -39.

Contreras, W., W. Valero, Edgard Thomson, M. Owen de C. y E. Barrios. 2007. Determinación de los esfuerzos de diseño de vigas lami- 
nadas de pino caribe (Pinus caribaea var. hondurensis), encoladas con adhesivo de isocianato (MDI). Revista Maderas. Ciencia y Tecnología. Concepción, Chile. 9 (3):2007.

Contreras, W. y M. Owen de C. 1.999. Análisis sobre la evolución de la madera laminada a través de su historia y su trascendencia para Venezuela en el siglo XX. Revista Forestal Latinoamericana. IFLA. Mérida, Venezuela. Rev. Fores. Latino., 1999, núm. 25:47-62.

Contreras, W., V. Cloquell Ballester y M. Owen de Contreras. 2005. La Madera. Productos Forestales. Industria Forestal. Conceptos y clasificación. Universidad Politécnica de Valencia. Universidad de Los Andes, Mérida, Venezuela. $81 \mathrm{pp}$.

Freas A.D., and M.L. Selbo. 1954. Fabrication and design of glued laminated wood. Structural members. Forest Products Laboratory. Wisconsin. USA. 235 pp.

León, W. y N. Espinoza de Pernía. 2002. Anatomía de la Madera. Universidad de Los Andes. Consejo de Publicaciones. Consejo de Desarrollo Científico, Humanístico y Tecnológico (CDCHT). Mérida, Venezuela. 412 pp.

Ninin, P. 2007. Efecto del reaserrado con la conicidad y curvatura sobre el rendimiento de materia prima, calidad de madera aserrada y la trabajabilidad del pino caribe de la orinoquia venezolana. Centro de Estudios Forestales y Ambientales de Postgrado (CEFAP). Facultad de Ciencias Forestales y Ambientales.
Universidad de Los Andes (ULA). Mérida, Venezuela. 215 pp.

Ross, R.J. 1985. Stress wave propagation in wood products. In: Proceedings, $5^{\text {th }}$ nondestructive testing of wood symposium; 1985 September; Pullman, WA: Washington State University. USA: 291-318.

Ross, R.J., K.A. Mc Donald, D.W. Green, y K.C. Schad. 1996. Relationship between log and lumber modulus of elasticity. Forest Products Journal, 47(2):89-92.

Smulski, S. 1997. Engineered Wood Products. A Guide for Specifiers, Designers and Users. PFS Research Foundation. Wisconsin. USA. 294 pp.

Trejo, E. 2006. Determinación de la proporción de madera juvenil en Pinus caribaea var. hondurensis en plantaciones de 10, 15 y 20 años (Chaguaramas Norte y Guayamure) bajo jurisdicción de la empresa Terranova de Venezuela. Centro de Estudios Forestales y Ambientales de Postgrado (CEFAP). Facultad de Ciencias Forestales y Ambientales. Universidad de Los Andes (ULA). Mérida, Venezuela. 301 pp.

Valero, S. 2001. Relación entre anatomía y propiedades físicomecánicas de la especie Tectona grandis, proveniente de los Llanos Occidentales de Venezuela. Centro de Estudios Forestales y Ambientales de Postgrado (CEFAP). Facultad de Ciencias Forestales y Ambientales. Universidad de Los Andes (ULA). Mérida, Venezuela. 245 pp. las propiedades mecánicas de vigas laminadas encoladas de madera de Pinus caribaea var. hondurensis. Madera y Bosques 16(3):23-35. 